So the problem of coupling and where it takes place is semantic. It depends on which set of (complete orthonormal) functions one chooses to expand the total field.

There still remains the important practical problem of how to fabricate DFB lasers so as to achieve the lowest possible threshold. We find by straightforward application of the coupled-mode formalism that the transition from a periodic waveguide to a uniform one is not essential to low-threshold operation in contrast to a finding by Wang. ${ }^{15}$ The unpumped sections of a periodic waveguide extending beyond the gain region merely act as passive reflectors. In the limit $\kappa L \gg 1$ ( $L$ is the length of the unpumped sections) their reflectivity approaches unity ${ }^{3}$ for frequencies $\omega$ such that $\Delta \beta(\omega) \leq \kappa$ (i。e, within the forbidden gap) and the laser threshold approaches zero. The presence of high losses in the unpumped region, which is the case in GaAs and dye lasers, tends to make the threshold similar to that which obtains in the geometry of Fig. 1(a) and which was treated by Kogelnik and Shank. This conclusion is supported by experimental data. ${ }^{8}$

In summary, the truncated Floquet-Bloch formalism is found to be fully equivalent to the conventional coupled-mode approach which has been used extensively in the analysis of Bragg optical devices (DFB lasers, periodic filters, and acoustic-optic interactions).
*Work supported by the Office of Naval Research.

${ }^{1} \mathrm{H}$. Koge Inik and C. V. Shank, Appl. Phys. Lett. 18, 152 (1971).

${ }^{2}$ H. Koge Inik and C. V. Shank, J. Appl. Phys. 43, 2327 (1972).

${ }^{3} \mathrm{H}$. Stoll and A. Yariv, Opt. Commun. 8, 5 (1973).

${ }^{4}$ A. Yariv, IEEE J. Quantum Electron. QE-9, 919 (1973).

5 J.R. Pierce, J. Appl. Phys. 25, 179 (1954).

${ }^{6} \mathrm{~W} . \mathrm{H}$. Louisell, Coupled Modes and Parametic Electronics (Wiley, New York, 1960).

${ }^{7}$ D.D. Schinke, R.G. Smith, E.G. Spencer, and M.F. Galvin, Appl. Phys. Lett. 21, 494 (1972).

${ }^{8}$ M. Naka mura, H.W. Yen, E. Garmire, S. Somekh, and

H. L. Garvin, Appl. Phys. Lett. 23, 224 (1973).

${ }^{9}$ D. F landers, H. Kogelnik, R. V. Schmidt, and C.V. Shank, Appl. Phys. Lett. 24, 194 (1 974).

${ }^{10}$ F.W. Dabby, A. Kestenbaum, and U.C. Paek, Opt.

Commun, 6, 125 (1972).

${ }^{11} \mathrm{~K}$. Sakuda and A. Yariv, Opt. Commun. 8, 1 (1973).

${ }^{12} \mathrm{R}$. Cordero (unpublished).

${ }^{13}$ S. Wang, J. Appl. Phys. 44, 767 (1973).

${ }^{14}$ R. Cordero and S. Wang, Appl. Phys. Lett. 24, 474 (1974).

${ }^{15}$ S. Wang, Wave Electronics 1, 31 (1975).

${ }^{16}$ S. Wang, Appl. Phys. Lett. 26, 89 (1975).

${ }^{17} \mathrm{~A}$. Yariv and H.W. Yen, Opt. Commun, 10, 120 (1974).

${ }^{18} \mathrm{D}$. Marcuse, Bell Syst. Tech. J. 48, 3187 (1969); also in

Light Transmission Optics (Van Nostrand-Reinhold, New York, 1972).

${ }^{19} \mathrm{~S}$. R. Chinn, IEEE J. Quantum Electron. QE-9, 574 (1973).

20 D.R. Scifres, R.D. Burnham, and W. Streifer, Appl. Phys. Lett. 25, 200 (1974).

\title{
Thermal blooming compensation using coherent optical adaptive techniques (COAT)
}

\author{
William B. Bridges* \\ California Institute of Technology, Pasadena, California 91125 \\ James E. Pearson \\ Hughes Research Laboratories, Malibu, California 90265 \\ (Received 2 January 1975; in final form 28 February 1975)
}

\begin{abstract}
Real-time compensation for thermally induced beam distortion has been demonstrated using a phase-adaptive aperture subdivided into 18 elements coupled with an 18-channel multidither servo system. The results were obtained using an argon ion laser and a nonlinear liquid cell placed in the near field of the transmitted beam. The system increased the peak beam irradiance by more than a factor of 4 at the highest power used and reduced the beamwidth by a factor of 5.4 from the uncorrected bloomed case. A comparison with predictions from existing theory is given.
\end{abstract}

PACS numbers: 42.65., 42.70.K, 42.68.D

High-power optical beams propagating through the atmosphere can experience distortion from two sources: turbulence (a linear process) and thermal blooming (a nonlinear process). Real-time compensation for the linear propagation problem, turbulence-induced phase distortion, has already been demonstrated using a selfadaptive optical phased array ${ }^{1-3}$ This article describes the successful use of such a $\operatorname{COAT}^{4}$ system to compensate for the nonlinear propagation problem of phase distortion induced in an absorbing liquid by a high-power laser beam.

The multidither-type COAT array used for these ex- periments is described elsewhere. ${ }^{5}$ In such a system the radiation from each element in the array is tagged with a different low-index sinusoidal phase modulation $\omega_{i}$ (the dithers). Radiation from the array passes through the disturbed propagation path to a remote target. A sample of the interference pattern is reflected by a small glint on the target (i.e., an area of higher reflectivity unresolved by the array). This reflected signal contains intensity-modulated components at all possible sums and differences of the phase modulation frequencies $\left(n \omega_{i} \pm n \omega_{j}\right)$. A bank of synchronous detectors fed by a simple intensity detector sorts out the signal components at the original frequencies and, after low- 


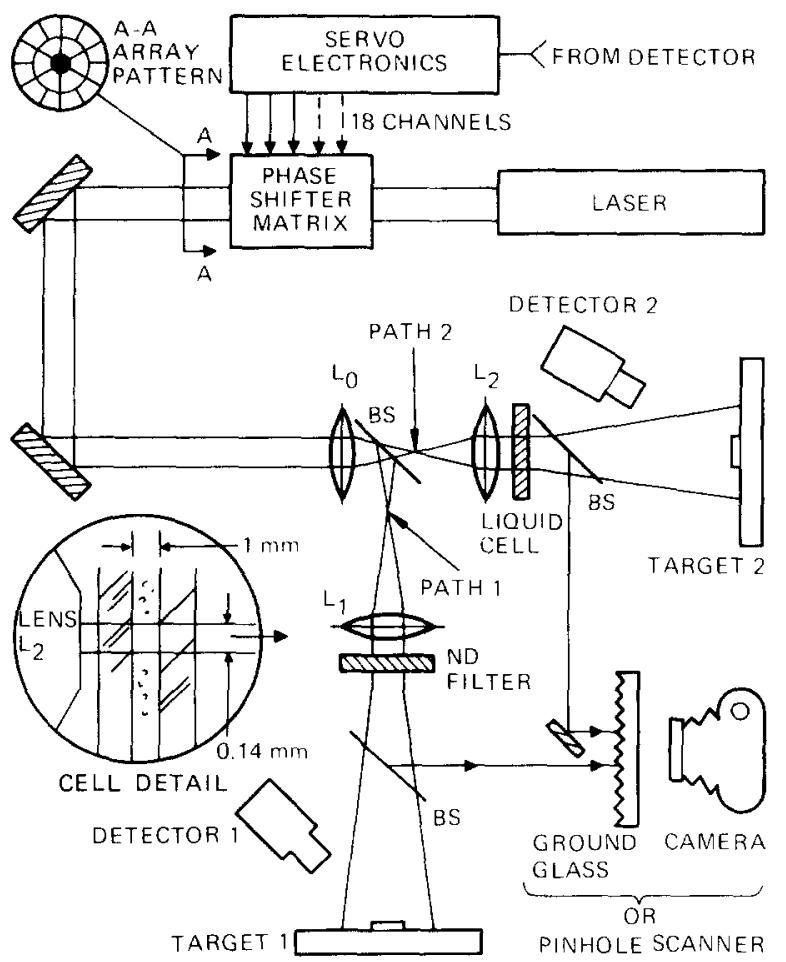

FIG. 1. Schematic arrangement of the optical paths. The two paths are identical except for the liquid cell in path 2 . The inset section A-A shows the spatial distribution of the 18-element phased array in two annular rings. The ground glass and camera could be replaced with a scanning point detector to profile the spatial distribution in both beams. The diameter of a diffraction-limited beam exiting either lens $L_{1}$ or $L_{2}$ was approximately $140 \mu \mathrm{m}$, as shown in the cell detail.

pass filtering, derives error signals that are fed to the optical phase shifter array used to perform the original tagging. The resulting multiple-loop "hill-climbing" servo system acts to maximize the irradiance on the target glint point. The maximum is reached when the pattern formed is identical to that of an ideally phased array.

The essential feature of this phasing scheme is that the array will be properly phased to produce the ideal pattern at the target even if a time-varying phase disturbance is introduced in the optical path, providing that the magnitude and speed of the disturbance are within the capability of the servo loop. It is not a priori obvious, however, that such a system will arrive at a unique or stable state if there is nonlinearity such as thermal blooming in the propagation path. ("Thermal blooming" is the name given to beam spreading caused by the power-dependent negative lens formed in an absorbing medium.)

The radiating aperture in this experiment was broken up into 18 contiguous planar elements arranged in two concentric rings as shown in the inset of Fig. 1. Eighteen equally spaced dither frequencies from 8 to 32 $\mathrm{kHz}$ were used with $20^{\circ}$ peak phase deviation. The phys ical arrangement shown in Fig. 1 employed two identi cal optical paths, each containing a demagnifying telescope (lens $\mathrm{L}_{0}$ with lens $\mathrm{L}_{1}$ or $\mathrm{L}_{2}$ ), a distance sufficient

to put the target in the far field of the telescope exit aperture, a scotchlite "glint" on the target board, and a detector to monitor the intensity reflected from the glint.

The control loop could be closed around either path by choosing the corresponding detector. Samples of the beam intensity distribution in both paths could be photographed or scanned as shown.

A nonlinear liquid cell was introduced in one of the paths in the near field of the transmitter. For these experiments, a $1-\mathrm{mm}$-thick cell containing iodine in a $90 \% \mathrm{CCl}_{4}-10 \%$ methanol solvent was used. The cell input window was at the effective transmitter plane; the laser beam confocal parameter was $10 \mathrm{~mm}$. The iodine concentration was adjusted to give $40 \%$ transmission of the $488-\mathrm{nm}$ radiation. The laser power was varied to provide different amounts of thermal blooming.

At low laser power levels, no thermal blooming was observed; essentially diffraction-limited beam intensity distributions were obtained at both targets, as shown in Figs. 2(a) and 2(d), independent of which detector was used to close the loop. This test demonstrates the optical quality of both paths and also the ability of the COAT system to phase the array properly in the absence of any propagation path disturbance.

NO COAT CORRECTION
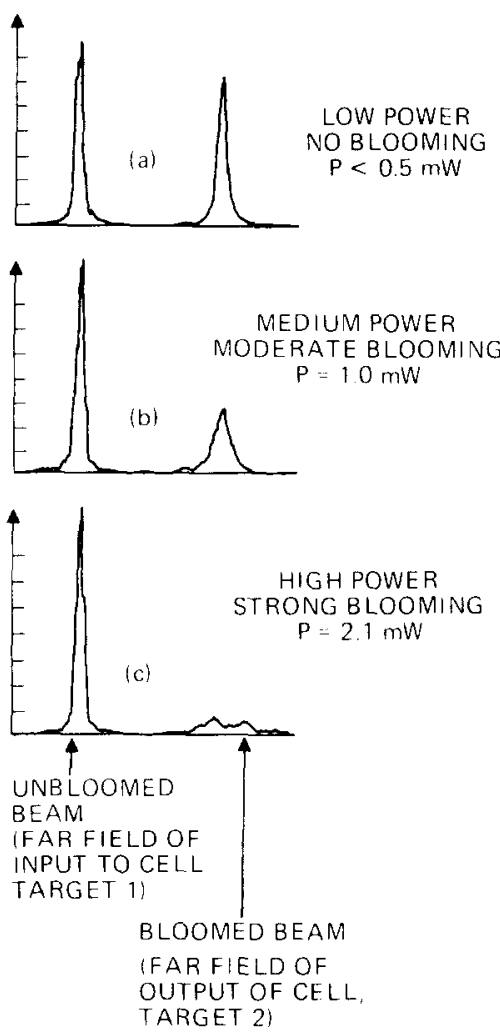

FIG. 2. Pinhole scans of the two beams taken at the location indicated in Fig. 1. In (a)-(c) the servo loop is closed around detector 1 so that the beam entering the cell is close to diffraction limited. In (d) $-(f)$ the loop is closed around detector 2 so that the beam exiting the cell is close to diffraction limited. The vertical scale in (f) is twice that of the other figures. 


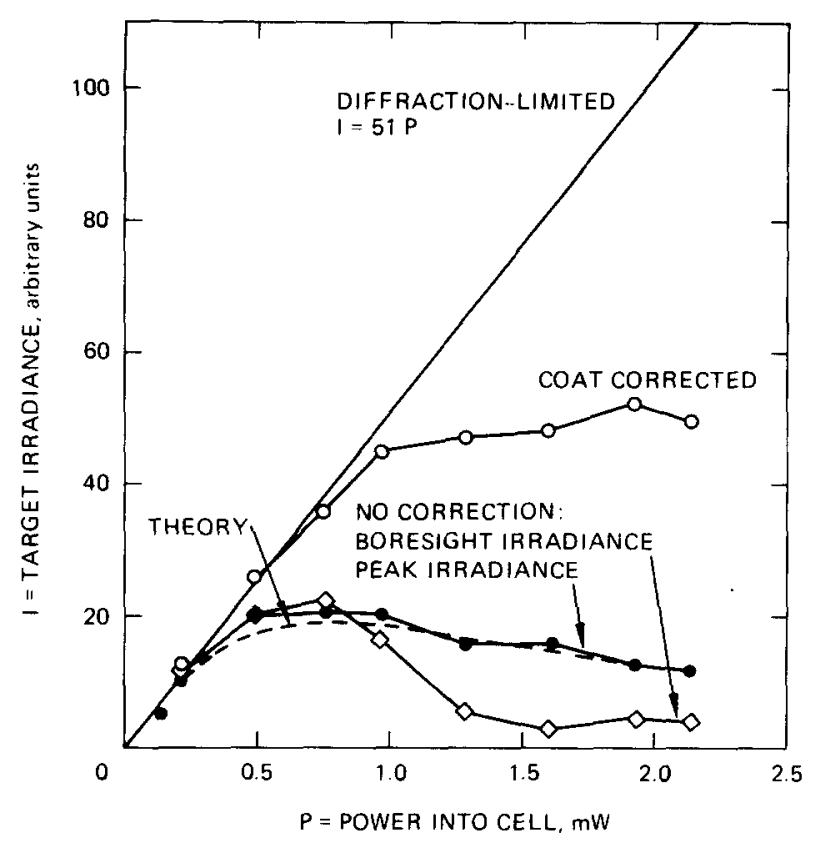

FIG. 3. Irradiance at the target as a function of the power incident on the absorbing cell. Open circles are for loop closed around detector 2 (COAT correction for blooming). Closed circles and diamonds are with loop closed around detector 1 (no COAT correction). The diamonds show the irradiance on the geometric boresight to the target defined by the glint position; the closed circles show the peak values of irradiance irrespective of where they occur within the distorted pattern. The dotted curve is the theoretical expression Eq. (6) with $A$ adjusted to $1.34 \mathrm{~mW}^{-1}$.

At higher power levels, the expected moderate thermal blooming was observed at target 2 when the COAT servo loop was closed around detector 1 as shown in Figs. 2(b) and 2(c). The beam remains diffraction limited entering the nonlinear cell, path 1 . If the servo loop is now closed around detector 2 , a well-formed beam occurs at target 2 while the beam at target 1 is distorted as shown in Figs. 2(e) and 2(f)。The COAT system has compensated for the phase distortion introduced by the nonlinear cell by predistorting the phase. distribution of the beam entering the cell. It is not surprising that the degree of distortion now at target 1 is approximately the same as that previously at target 2 since the phase distribution generated by the COAT system should be the complex conjugate of the phase distortion introduced by the thin cell. It is also not surprising that the amplitudes of the COAT-corrected beams in Figs. 2(e) and 2(f) are not as great as the corresponding undistorted beams in Figs. 2(b) and 2(c). The 18-element array of planar mirrors can only make a step-wise approximation with five resolution elements across the aperture to the roughly quadratic phase distortion introduced by the medium. The residual distortions cause the "missing energy to go into grating lobes (axial rings) which can be seen in Figs. 2(e) and 2(f).

The quantitative improvement in irradiance at the target is shown in Fig. 3. The solid straight line is the irradiance expected in the absence of nonlinear effects. The two lowest solid curves show the measured irradi- ance at the target when thermal effects distort the beam. Since the distortion breaks the beam up into many "blobs", both the irradiance on the boresight and the peak irradiance anywhere in the beam are shown. As shown by the upper solid curve, the COAT system extends the range of near-ideal performance by a factor of 2 or 3 in power, and makes an improvement in the peak beam irradiance of $2-4$ times depending on the power level.

We may estimate the power level at which the COAT correction to the quadratic phase term introduced by the thermal lens begins to fail. The best that each array element can do is to correct the average phase over that element. When the rms value of the difference between the actual phase and this average approaches $\frac{1}{8} \lambda$ or so for any element, then the correction will be less than ideal. For the negative lens, this occurs first at the outermost ring of the array. We may approximate the actual situation by considering an $N$-element linear array $(N=5$ for the 18 -element array illustrated in Fig. 1). The rms phase error on the outermost elements is then

$$
\phi_{e}^{\mathrm{rms}}=(2 k / \sqrt{3} N)\left(1-2 / N+16 / 15 N^{2}\right)^{1 / 2},
$$

where $\beta$ is the uncorrected value of phase error at the edge of the array. If $\phi_{a}^{\mathrm{rms}}$ must be less than $\frac{1}{8} \lambda$ then $\beta$ must be less than about $0.7 \lambda$ for $N=5$ 。

To relate $\beta$ to the laser power $P$ we use the formalism of Gordon et $a l .{ }^{6}$ appropriately modified for larger absorption coefficients and cooling at the end walls of the cell. ${ }^{7}$ The far-field beam spreading in an initially collimated Gaussian beam passed through a lossy liquid cell of length $l$ is

$$
\theta_{\text {therm }}=2 \alpha l P[1+\exp (-\alpha l)] \frac{d n}{d T}\left(1.04 w_{0} k \pi^{3}\right)^{-1}
$$

where $\theta_{\text {therm }}$ is the full angle of the spread beam, $P$ is the incident beam power in $W, \alpha$ is the absorption coefficient in $\mathrm{cm}^{-1}, d n / d T$ is the temperature coefficient of the refractive index in ${ }^{\circ} \mathrm{K}^{-1}, w_{0}$ is the Gaussian beam radius in $\mathrm{cm}$, and $k$ is the thermal conductivity in cal $\mathrm{cm}^{-1} \mathrm{sec}^{-1}{ }^{\circ} \mathrm{K}^{-1}$. It is convenient to compare this angle with the diffraction angle of a collimated beam of the same diameter,

$$
\theta_{\text {diff }}=2 \lambda / \pi w_{0}
$$

by forming the ratio

$$
\frac{\theta_{\text {therm }}}{\theta_{\text {diff }}}=\frac{\alpha l}{1.04 \pi^{2}}[1+\exp (-\alpha l)] \frac{d n}{d T}(k \lambda)^{-1} P \equiv A P .
$$

This ratio is independent of the beam dimensions, and thus may be accurately measured without knowing the precise beam diameter. It is also easy to show that $\beta$ in Eq. (1) (in wavelengths) is given by

$$
\beta=(1 / 2 \pi) \theta_{\text {therm }} / \theta_{\text {diff }},
$$

so we have the desired relation $\beta(P)$ and hence $\phi_{e}^{\text {rma }}(P)$ by combining Eq. (4) with (5) and (1)。

Before carrying through the comparison for the COAT-corrected curve of Fig. 3, it is useful to compare the predictions of $\mathrm{Eq}$. (4) with the uncorrected thermally bloomed beam results shown on Fig. 3. The 
irradiance reaching a pinhole detector in the target plane will be reduced by the thermal beam spreading and can be expressed as

$$
I_{\mathrm{tarket}}=B P\left(\frac{\theta_{\text {disf }}^{2}+\theta_{\text {therm }}^{2}}{\theta_{\mathrm{dif}}^{2}}\right)^{-1}=\frac{B P}{1+(A P)^{2}},
$$

where $A$ is defined by Eq. (4) and $B$ is a proportionality constant expressing the experimental propagation path geometry. The dotted curve on Fig。 3 is a plot of Eq. (6) with $A$ adjusted to $1.34 \mathrm{~mW}^{-1}$ to give a best fit to the experimental data. The parameter $B$ has been fixed at the value giving the straight line when $A=0$. We see that the experimental curve fits the form predicted by theory quite well.

We may also compare these experimental values of $A$ with those given directly from $\mathrm{Eq}$. (4). The parameters of the iodine $/ \mathrm{CCl}_{4}\left(10 \% \mathrm{CH}_{3} \mathrm{OH}\right)$ cell are

$$
\begin{aligned}
& \exp (-\alpha l)=0.40, \\
& \frac{d n}{d T}=5.6 \times 10^{-4}{ }^{\circ} \mathrm{K}^{-1}, \\
& k=2.6 \times 10^{-4} \mathrm{cal} \mathrm{cm}^{-1} \mathrm{sec}^{-1}{ }^{\circ} \mathrm{K}^{-1}, \\
& \lambda=0.488 \times 10^{-4} \mathrm{~cm},
\end{aligned}
$$

giving $A=5.5 \mathrm{~mW}^{-1}$, about 4 times larger than the observed value. We have considered several factors which might explain or justify the smaller-than-predicted value of blooming:

(i) The transverse beam distribution radiated by the COAT-phased array is not the untruncated Gaussian assumed by Gordon et al. ${ }^{6}$ or the $J_{0}$ Bessel approximation used by Dabby et $a l,{ }^{7}$ but rather is a Gaussian truncated at the $-3 \mathrm{~dB}$ points with a central obscuration of $20 \%$. Simple estimates of the magnitude of this effect, ${ }^{8}$ however, indicate that such corrections are small, not a factor of 4 .

(ii) Natural convection induced by the deposited heat could also partly reduce the amount of blooming. The thermal diffusion time $t_{c}$ (Ref。6) is only $15 \mathrm{msec}$ in our cell while the observed transit time of motes across the beam diameter is at least 10 times slower. From this we would conclude that convection effects would be negligible, however, the uncorrected bloomed beam was observed to be somewhat nonaxisymmetric as those illustrated in Ref.9, and deflected slightly downward, indicating a vertical temperature gradient.

(iii) An independent thermal blooming experiment ${ }^{8}$ was also conducted using different lasers ( $\mathrm{He}-\mathrm{Cd}$, $\mathrm{He}-$ $\mathrm{Ne})$, a different cell size $(1 \times 1 \times 2 \mathrm{~cm})$, different dyes, and different solvents (methyl alcohol, benzene) with similar results: a factor of 2-3 times less blooming than predicted.

(iv) The amount of thermal blooming indicated in Fig. 2 of Ref. 9 appears to indicate a factor of 4 in $\theta_{\text {tot al }} /$ $\theta_{\text {diff }}$ (if the photographs are the same scale). The experimental parameters given in Ref. 9 predict a factor of 24 , however.

We are unable to account adequately for the lack of agreement between theory and experiment for the uncorrected bloomed beam. However, if we proceed and find $\beta(P)$ by combining Eqs. (4) and (5) and assume that the correction will begin to fail when $\beta \simeq 0.7 \lambda$, then we find that $P_{\text {crit }}=4.4 A^{-1}$. This gives a value of $P_{\text {crlt }}=0.8$ $\mathrm{mW}$ when $A=5.5 \mathrm{~mW}^{-1}$. Figure 3 indicates a value of $0.75 \mathrm{~mW}$ for $P_{\text {crit }}$, in excellent agreement with predictions. We would expect that $P_{\text {crit }}$ could be increased proportionally to the number of elements across the diameter of the array.

The important result of this experiment is that the closed-loop correction provided by the COAT system results in a stable situation when the nonlinear medium is in the near field of the radiation aperture. That is, the focusing provided by the COAT system does not strengthen the negative thermal lens, which would require more correction and so forth, so that a continual ly evolving situation results. Furthermore, we observe that if the nonlinear cell is mechanically jiggled, thus perturbing the position of the negative lens with respect to the optical beam that formed it, the COAT system continues to maintain a diffraction-limited beam on the glint point. Thus some turbulence and thermal blooming can be compensated at the same time. The servo-loop time constant in this system is approximately $1 \mathrm{msec}$.

Additional work is now in progress to determine the effects of forced convection in both flowing liquid and flowing gas cells. The effectiveness of adaptive phase compensation when the nonlinear medium is not concentrated in the transmitter near field but instead is distributed along the propagation path from the near-field region to the far-field (focal plane) region is also being studied. Because COAT corrects via phase changes at the transmitter plane, it is expected to have less effect on phase distortions which occur in the far field since these transform to amplitude variations in the transmitter plane.

The authors are indebted to W.P. Brown, Jr., N. George, T. R. O'Meara, and J.R. Whinnery for valuable discussions and to F.C.Trimble for his able technical assistance. The COAT system used in these experiments was built with contract support from the Defense Advanced Research Projects Agency monitored through the Rome Air Development Center. Some of the later work (Ref. 8) was accomplished while one of the authors (WBB) was at Caltech as a Sherman Fairchild Distinguished Scholar. The support of Caltech and the Sherman Fairchild Foundation is gratefully acknowledged.

\footnotetext{
*Permanent address: Hughes Research Laboratories, Malibu, Ca, 90265 .

${ }^{1}$ W. B. Bridges, P.T.Brunner, S. P. Lazzara, T.A. Nussmeier, T.R. O'Meara, J.A. Sanguinet, and W. P. Brown, Jr., Appl. Opt. 13, 291 (1974).

${ }^{2}$ W. B. Bridges, S. Hansen, L. A. Horwitz, S. P. Lazzara, T.R. O'Meara, J.E. Pearson, and T.J. Walsh, J. Opt. Soc. Am. 64, 541 (1974).

${ }^{3}$ J. E. Pearson, W. B. Bridges, L. S. Horwitz, T.J. Walsh, and R.F. Ogrodnik, Paper ThB5, OSA Topical Meeting on Optical Propagation Through Turbulence, Boulder, Colo., 1974 (unpublished).

4The acronym COAT, for coherent optical adaptive techniques,
} 
has been used generally to denote optical phased arrays that obtain their phasing information from the target and propagation path in a closed-loop fashion.

${ }^{5}$ W. B. Bridges, J.E. Pearson, S. Hansen, L. S. Horwitz, R. M. Kubo, S. P. Lazzara, T.R. O'Meara, and T.J. Walsh, RADC Technical Reports RADC-TR-384, 1973; RADC-TR74-38, 1973; and RADC-TR-74-108, 1974 (unpublished).
${ }^{6}$ J. P. Gordon, R. C.C. Leite, R.C. Moore, S. P.S. Porto, and J.R. Whinnery, J. Appl. Phys. 36, 3 (1965).

${ }^{7}$ F.W. Dabby, R.W. Boyko, C.V. Shank, and J.R. Whinnery, IEEE J. Quantum Electron. QE-5, 516 (1969).

${ }^{8}$ W. B. Bridges (unpublished).

${ }^{9}$ J.R. Whinnery, D. T. Miller, and F. Dabby, IEEE J. Quantum Electron. QE-3, 382 (1967).

\title{
A method of utilizing lithium-induced nuclear reactions in the determination of carbon, nitrogen, and oxygen in thin films
}

\author{
C. Cardinal, C. Brassard, J. Chabbal, L. Deschenes, J. P. Labrie, and J. L. L'Ecuyer \\ Laboratoire de Physique Nucléaire, Université de Montréal, Montréal, Canada
}

\begin{abstract}
Marc Leroux*
Centre de Recherches de l'Energie, I.N.R.S., Université du Québec, Varennes, Québec, Canada (Received 17 February 1975)

A method of analysis of oxygen, nitrogen, and carbon in thin samples involves the detection of charged particles from nuclear reactions induced by a $7-\mathrm{MeV}$ lithium- 6 beam. The particle detection with a telescope counter near $0^{\circ}$ takes full advantage of highly exothermic reactions. The sensitivity is of the order of $10^{-3} \mu \mathrm{g} / \mathrm{cm}^{2}$. A preliminary study of the contaminants introduced during the sputtering of niobium is presented.
\end{abstract}

PACS numbers: $61.70 . \mathrm{W}, 82.80 . \mathrm{F}$

The direct observation of charged particles emitted from low-energy ion-induced nuclear reactions has proved to be a powerful tool for thin solid film studies. This technique is complementary to backscattering; background-free detection of light contaminants in a matrix of heavy elements is possible below the Coulomb barrier. Both methods are also inherently capable of yielding depth profiles without recourse to sample erosion.

Various groups have reported analyses of carbon, nitrogen, and oxygen obtained with low-energy $(<2$ $\mathrm{MeV})$ proton, ${ }^{1,2}$ deuteron, ${ }^{3-5}$ triton, ${ }^{5}$ and helium ${ }^{6}$ beams. These analyses involved the detection of the particles emitted at backward angles. Particle identification was accomplished through insertion of absorbers between the target and the detector.

In order to meet the needs of modern technology, greater sensitivity and better depth resolution are required. The heavy-ion-induced exothermic reactions offer such a possibility, in the cases where the lowlying states appear in a completely background-free region of the spectrum. The large stopping power of heavy ions represents a great asset when the emitted particle is detected at forward angles. The beam can be stopped in front of the detector and the favorable kinematics at $0^{\circ}$ permit large counting solid angles.

Results obtained with the lithium-6-induced reactions ${ }^{14} \mathrm{~N}\left({ }^{6} \mathrm{Li}, \alpha\right){ }^{16} \mathrm{O},{ }^{12} \mathrm{C}\left({ }^{6} \mathrm{Li}, d\right){ }^{16} \mathrm{O}$, and ${ }^{16} \mathrm{O}\left({ }^{6} \mathrm{Li}, d\right){ }^{20} \mathrm{Ne}$ are reported herein. Measurements were made on niobium targets, sputtered on copper foils subsequently dis solved in nitric acid. The targets were therefore selfsupporting with thicknesses of the order of $1.0 \mathrm{mg} / \mathrm{cm}^{2}$.
The geometry of the detection system is shown in Fig. 1. The beam is stopped in a circular tantalum disk of sufficient thickness to absorb all possible reaction products. This stopper covers the center of the detector-sensitive area, leaving an effective solid angle of $5 \times 10^{-2} \mathrm{sr}$ for detection. The elastically scattered beam is absorbed in a stack of nickel foils of 11 $\mathrm{mg} / \mathrm{cm}^{2}$ total thickness.

The determination of the atomic number $Z$ and of the mass number $A$ of the detected particles is an essential part of the system. The reaction products are identified by a conventional $\Delta E-E$ detector telescope system. The principle of operation is that the range-energy relationship of light nuclei can be approximated by the empirical power-law formula ${ }^{7} R=\alpha \mathcal{E}^{b}$, where $R$ is the range of the particle of energy $\varepsilon$ and $\alpha$ is a constant characterizing the particle. The exponent $b$ is nearly constant

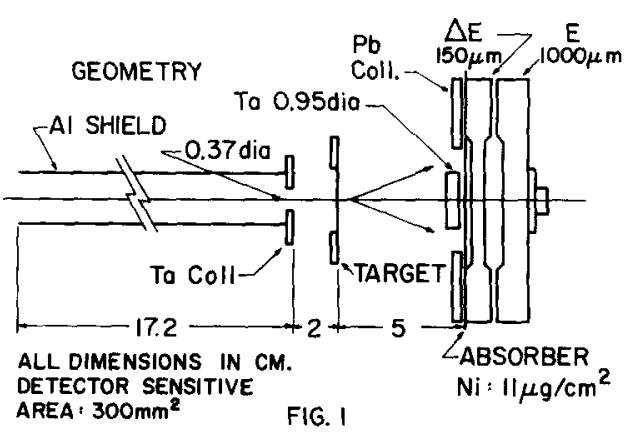

FIG. 1. Geometry of detection, showing the target and the tantalum stopper positions relative to the counter telescope. 